\title{
Modification of $\mathrm{O}$ and $\mathrm{CO}$ binding on $\mathrm{Pt}$ nanoparticles due to electronic and structural effects of titania supports
}

Tom Ellaby, ${ }^{1}$ Ludovic Briquet, ${ }^{2}$ Misbah Sarwar, ${ }^{2}$ David Thompsett, ${ }^{2}$ and Chris-Kriton Skylaris ${ }^{1}$ a)

${ }^{1)}$ Department of Chemistry, University of Southampton

2) Johnson Matthey Technology Centre

(Dated: 20 August 2019)

Metal oxide supports often play an active part in heterogeneous catalysis by moderating both the structure and the electronic properties of the metallic catalyst particle. In order to provide some fundamental understanding on these effects, we present here a DFT investigation of the binding of $\mathrm{O}$ and $\mathrm{CO}$ on Pt nanoparticles supported on titania (anatase) surfaces. These systems are complex and in order to develop realistic models here we needed to perform DFT calculations with up to $\sim 1000$ atoms. By performing full geometry relaxations at each stage, we avoid any effects of "frozen geometry" approximations. In terms of the interaction of the Pt nanoparticles with the support, we find that the surface deformation of the anatase support contributes greatly to the adsorption of each nanoparticle, especially for the anatase (001) facet. We attempt to separate geometric and electronic effects, and find a larger contribution to ligand binding energy arising from the former. Overall, we show an average weakening (compared to the isolated nanoparticle) of $\sim 0.1 \mathrm{eV}$ across atop, bridge and hollow binding sites on supported $\mathrm{Pt}_{55}$ for $\mathrm{O}$ and $\mathrm{CO}$, and a preservation of site preference. Stronger effects are seen for $\mathrm{O}$ on $\mathrm{Pt}_{13}$, which is heavily deformed by anatase supports. In order to rationalise our results and examine methods for faster characterisation of metal catalysts, we make use of electronic descriptors, including the d-band centre and our electronic density based descriptor. We expect that the approach followed in this study could be applied to study other supported metal catalysts.

\section{INTRODUCTION}

Metal oxides find broad use as supports for metal nanoparticle catalysts ${ }^{1,2}$ in industrial applications, including ammonia synthesis ${ }^{3}$, the Fischer-Tropsch reaction ${ }^{4}$, the water-gas shift (WGS) $)^{5}$ and steam reforming processes ${ }^{6}$. Their high thermal stability and typically strong interaction with metal nanoparticles (therefore high binding strength, to anchor the nanoparticle) $)^{7}$ are two of the main reasons for their use. Strong interactions also lead to a modification of the electronic properties of the nanoparticles, which can be exploited in catalyst design.

Titania, or titanium dioxide $\left(\mathrm{TiO}_{2}\right)$, has been used as a support for Pt and Co nanoparticles to catalyse the $\mathrm{WGS}^{8}$ and Fischer-Tropsch reactions ${ }^{9}$, respectively, and finds use in photocatalysis $^{10,11}$ and other chemical applications ${ }^{12}$.

There are three predominant phases of titania: rutile, anatase and brookite. The latter is significantly less stable than the other two and has fewer applications because of it ${ }^{13}$. Rutile is known to be the more stable of the two at ambient temperatures in bulk ${ }^{14}$, although difficulties in experimental work ${ }^{15}$ mean a wide range of enthalpy change between rutile and anatase have been reported. Theoretical calculations with DFT do not resolve this issue, and can also predict either as the more stable phase, depending on the choice of basis set and functional used ${ }^{16}$. It is understood, however, that the surface energy contributions to the total energy are lower for anatase compared to rutile ${ }^{17}$, which leads to smaller (nano)particles favouring the anatase phase. In any case, the difference in energy between the two phases is small, and anatase is stable up to $800{ }^{\circ} \mathrm{C}^{13}$.

\footnotetext{
a)Electronic mail: c.skylaris@ soton.ac.uk
}

The size and shape of metal nanoparticles can have a significant effect on their chemical properties ${ }^{18}$, including their catalytic properties. Small nanoparticles in particular can have vastly different properties compared to the bulk ${ }^{19,20}$.

For heterogeneous catalysis, the Sabatier principle suggests that the binding energies to the catalyst of the reactants and products of a given reaction should be neither too strong or too weak in order to maximise the turnover frequency (TOF) ${ }^{21}$. Rather than calculate the binding energies of the reactants and products in all their possible configurations for a given reaction, the binding energies of relevant smaller ligands, such as $\mathrm{O}, \mathrm{OH}, \mathrm{CO}$, can be used to predict the TOF instead ${ }^{22,23}$. For example, the adsorption energy of nitrogen on a catalyst surface can be used to predict the TOF of ammonia synthesis ${ }^{24}$. Depending on the reaction, this can be remarkably accurate, and reduces the complexity of the theoretical calculations substantially.

Previous work in the literature has focused on Pt on titania as a catalyst for the WGS reaction at low temperatures ${ }^{25}$. This reaction produces $\mathrm{H}_{2}$ and $\mathrm{CO}_{2}$ from carbon monoxide and water, and while a faster rate of conversion is achieved at high temperature, the equilibrium also favours the reactants at high temperature. Therefore, typical industrial practice is to employ a two stage process: a high temperature shift followed by a low temperature shift. The catalysts for these two stages are different, mainly due to the requirement for increased thermal stability for the higher temperature reaction. Metal oxide supported metal nanoparticles tend to become more mobile at higher temperatures, causing them to agglomerate leading to a loss of activity. Kwon et al. ${ }^{25}$ showed that a combination of partially reduced graphite oxide, $\mathrm{TiO}_{2}$ and $\mathrm{Pt}$ nanoparticles effectively catalysed the WGS at $280{ }^{\circ} \mathrm{C}$ with $80 \%$ conversion of $\mathrm{CO}$, while resisting oxidation. The latter point is important since a potential application of the WGS reaction is as a feed for a PEM fuel cell, which needs to operate in oxidising 
conditions ${ }^{26}$.

Both the geometric and electronic properties are affected by the support material, even for weakly interacting metals and supports like Pt on graphene ${ }^{27}$. A graphene support weakens the adsorption energies of $\mathrm{O}$ and $\mathrm{CO}$ on $\mathrm{Pt}$, although this effect is diminished as the size of the nanoparticle increases. Theoretical calculations show this contribution to the ligand binding strength on nanoparticles larger than $\sim 2 \mathrm{~nm}$ is negligible.

In this work, we investigate, via density functional theory (DFT) calculations, how the support affects the binding energies of oxygen and carbon monoxide to Pt nanoparticles, as well as nanoparticle stability on different anatase facets. We use existing descriptors and models to predict how this choice of support might ultimately affect catalytic properties. We also test our previously introduced descriptor ${ }^{28}$, based on electrostatic potential and electron density, to understand its applicability to supported systems.

\section{METHODS}

We have performed DFT calculations with the ONETEP ${ }^{29}$, and CASTEP ${ }^{30}$ codes. ONETEP is a linear scaling DFT code capable of simulating large systems. It employs highly localised non-orthogonal generalised Wannier functions (NGWFs) and a basis set of periodic sinc (psinc) functions, which are equivalent to plane waves. The NGWFs are centred on atomic sites, and are optimised on-the-fly to provide near complete basis set accuracy, equivalent to a plane wave approach with the same kinetic energy cut-off. In the ONETEP implementation of ensemble DFT (EDFT) ${ }^{31}$, which was used in order to accurately model our metallic systems, the inner loop minimises the free energy with respect to the Hamiltonian matrix elements, while the outer loop minimises a projected functional with respect to the NGWFs ${ }^{32}$.

For our calculations here, EDFT with a Fermi-Dirac smearing of $0.1 \mathrm{eV}$ (electronic temperature of $1160 \mathrm{~K}$ ) was used. Core electrons are modelled with the projector augmented wave (PAW) method ${ }^{33}$ using PAWs from the GBRV set ${ }^{34}$. The configuration for the explicitly modelled valence electrons in $\mathrm{Pt}$ is $5 \mathrm{~d}^{9} 6 \mathrm{~s}^{1}$. The kinetic energy cut-off for the basis set was set at $850 \mathrm{eV}$ and NGWF radii of 9.0 Bohr were used. The calculations were performed at the $\Gamma$-point only, in cells with a $15.0 \AA$ vacuum padding in non-periodic directions.

CASTEP is a more conventional plane wave DFT code well suited to solid state systems. All CASTEP calculations we performed used the density mixing scheme with a Gaussian smearing of width $0.1 \mathrm{eV}$, ultrasoft pseudopotentials from the same GBRV set as the PAWs used with ONETEP, and a kinetic energy cut-off of $500 \mathrm{eV}$.

For all calculations (in both codes), the exchangecorrelation energy was described within the generalised gradient approximation (GGA) using the revised Perdew-BurkeErnzerhof (rPBE) functional ${ }^{35,36}$. The anatase unit cell, taken from the crystallography open database (originally from Rezaee et $a l .{ }^{37}$ ) was relaxed in bulk using the lattice optimisation procedure available in CASTEP. This unit cell was then cleaved to expose the different facets, using terminations that produced the correct stoichiometry and ensuring both the top and bottom of the slab were the same.

DFT geometry relaxations were performed using CASTEP. These involved slab relaxation and thickness convergence testing, and nanoparticle deposition and oxygen binding calculations on $\mathrm{Pt}_{13}$ and $\mathrm{Pt}_{55}$. Single point energy calculations were performed on the relaxed geometries using ONETEP which were then used to determine properties such as the projected/local density of states (PDOS/LDOS). Slab thickness convergence testing was performed in CASTEP by adding extra layers of atoms in such a way as to: a) keep the same surface termination, and b) preserve the stoichiometry of the system.

For calculations of all binding energies reported in this work we have used Equation 1

$$
E_{\text {binding }}=E_{a+b}-\left(E_{a}+E_{b}\right)
$$

where the $a$ and $b$ in subscript refer to the separated, isolated systems, with $\mathrm{a}+\mathrm{b}$ referring to the combined system. The systems $a, b$, and $a+b$ are either fully or partially geometry relaxed, as follows: clean anatase slabs have their bases fixed (bottom 2 layers); isolated nanoparticles have no constraints; slabs with supported nanoparticles have the same constraints as the separated systems (i.e. fixed anatase base layers and no constraints on the nanoparticle); anatase supports are fixed (but the nanoparticle is not) for oxygen and carbon monoxide ligand binding calculations; isolated oxygen and carbon monoxide molecules are fully relaxed.

For the oxygen binding calculations, $E_{a}$ is taken as half the energy of an isolated oxygen molecule, performed with spin polarisation (to correctly describe the triplet state).

\section{A. Descriptors}

The d-band centres were calculated as the first moment or mean energy of the d-band, as per ${ }^{38}$. The d-bands themselves are calculated using the inbuilt local and angular momentum projected density of states (LPDOS) method available in ONETEP. Validation of these LPDOS calculations was performed using small systems by comparing against CASTEP using OPTADOS ${ }^{39}$. In both cases, the same projection scheme was used, which projects the electronic density onto pseudoatomic orbitals of the desired angular momentum. For all systems where both methods could be used, the resulting PDOS plots were close to identical, as shown in Figure 1 the supplementary material.

Our electronic descriptor, first described in our earlier paper $^{28}$, is calculated by projecting the electronic density onto an isosurface of the electrostatic potential of the system. Volumetric data of these two properties are generated by ONETEP properties calculations and then an external python script is used to extract the density on an isosurface at the desired locations. One thing to note is that the electrostatic potential is defined to an arbitrary constant, which in plane-wave DFT is usually based on values in the pseudopotentials, and can be affected by many things in any given calculation. Because of 
this, care must be taken when comparing different systems, as we do in this work. In order to make the isosurfaces equivalent between systems, we take a set of points a fixed (small) distance away from each Pt atom in the system, average the electrostatic potential at those points and shift the electrostatic potential of the whole system such that the average of these points is the same as the average obtained for an isolated $\mathrm{Pt}$ atom. The assumption here is that the electrostatic potential inside the pseudopotential region (hence the small distance chosen) will be largely unaffected by the rest of the system, and so can be taken as equivalent for each of our different systems.

\section{RESULTS \& DISCUSSION}

\section{A. Titania Surfaces}

The anatase unit cell contains $4 \mathrm{TiO}_{2}$ units. Each Ti atom is bound to six $\mathrm{O}$ atoms via four equatorial and two apical bonds of $1.932 \AA$ and $1.979 \AA$, respectively, as measured via neutron diffraction ${ }^{40}$.

Our anatase supports are constructed from a DFT relaxed bulk, with lattice parameters of $a=b=3.820(+1.0 \%)$ and $c=9.812(+3.3 \%)$, where the values in parentheses are the percentage difference from experimental data ${ }^{40}$. An overestimation of $2.5 \%$ for the $c$ lattice parameter was reported by Lazzeri et al. ${ }^{41}$ when using the (non-revised) PBE functional.

In this work, we focus on three low index facets: (100), (001) and (101) which are the most prevalent and are summarised below:

(100) While the (100) facet does not have a surface energy much greater than the most stable (101) facet, the (100) is not present in the Wulff construction of the anatase crystal ${ }^{41}$. This facet is made up of predominantly fivefold coordinated Ti and twofold coordinated $\mathrm{O}$, although some bulk coordinated (threefold for $\mathrm{O}$ sixfold for $\mathrm{Ti}$ ) atoms of both species are also exposed in the grooves that are present.

(001) This facet is energetically unfavourable compared to both the (100) and (101) facets. However, it is present in the Wulff construction. The surface is comprised of only fivefold coordinated Ti and twofold coordinated $\mathrm{O}$.

(101) This facet is the most stable and most predominant, accounting for more than $94 \%$ of the Wulff construction surfaces $^{41}$. As with the (100) surface, there is some twofold coordinated $\mathrm{O}$ and fivefold Ti, but there is a larger fraction of bulk coordinated atoms (compared to (100)) in this case.

TABLE I. Surface energies of different anatase facets. This work is compared to that of Lazzeri et al. ${ }^{41}$, denoted with $*$ (converted from $\mathrm{Jm}^{-2}$ ).

\begin{tabular}{lccc}
\hline \hline Surface & $(100)$ & $(001)$ & $(101)$ \\
\hline $\mathrm{E}_{\text {surf }}\left[\mathrm{eVA}^{-2}\right]$ & 0.0262 & 0.0533 & 0.0192 \\
$\mathrm{E}_{\text {surf }}^{*}\left[\mathrm{eVA}^{-2}\right]$ & 0.0362 & 0.0612 & 0.0305 \\
\hline \hline
\end{tabular}

The ordering of the surface energies, given in Table I, is the same as was found by Lazzeri et al. ${ }^{41}$. There appears to be a fairly rigid shift of $\sim 0.01 \mathrm{eVA}^{-2}$ between our results and theirs, although we find the (101) surface to be relatively even more stable than the other two, if only slightly. This may be due to the thickness of the slab used. We found a convergence of surface energy with respect to slab thickness to be quite slow for the (101) facet, while the others did not suffer as much from this. The (101) still showed a significant increase in stability with increasing thickness up to $\sim 13 \AA$ (4 Ti layers). The other facets were converged to similar levels (1-2\% change with each added layer) by $\sim 10.0 \AA$ ( 1 unit cell length for (001), 3 for (100)).

\section{Deposited Pt Nanoparticles}

Perhaps the most striking observation we see in Table II is that the (001) facet binds the Pt nanoparticles much more strongly than the (100) and (101) anatase surfaces. The very strong binding of $\mathrm{Pt}_{13}$ to the anatase $(001)$ facet $(-4.86 \mathrm{eV}$ per interfacial $\mathrm{Pt}$ atom) is due to a combination of effects. Firstly, the anatase (001) facet is known to have a higher reactivity than the other facets in several applications (see review by Vittadini et $a l^{42}$ and references therein). This is attributed to its increased number of undercoordinated atoms, especially oxygen, compared to the other low index facets. Secondly, we observe in our geometry relaxations large distortions/reconstructions across the anatase surface upon deposition of the nanoparticle, centred around the deposition site. In the immediate vicinity of the nanoparticle, both $\mathrm{Ti}$ and $\mathrm{O}$ atoms are displaced, though the bridging $\mathrm{O}$ atoms are most affected, with the closest all forming a bond with a Pt atom, in some cases breaking Ti-O bonds to do so. These reconstructions contribute significantly to the calculated binding energy: performing this calculation with a fully fixed anatase surface weakens the binding energy to $-4.01 \mathrm{eV}(-1.34 \mathrm{eV}$ per interfacial $\mathrm{Pt}$ atom), a reduction in binding strength of more than $10 \mathrm{eV}$. The nanoparticle on the (001) surface is also more distorted compared to the (100) and (101), with Pt-Pt coordination lower overall. The (101) and (100) anatase facets bind $\mathrm{Pt}_{13}$ similarly to each other, with a binding strength of -1.24 $\mathrm{eV}$ and $-1.29 \mathrm{eV}$ per interfacial $\mathrm{Pt}$ atom, respectively.

TABLE II. Binding energies of cuboctahedral $\mathrm{Pt}_{13}$ and $\mathrm{Pt}_{55}$, and icosahedral $\mathrm{Pt}_{55}$ on different anatase facets. The Miller indices in the leftmost column indicate which facet of the nanoparticle is in contact with the support. Energies given are for the most favourable deposition site found (where multiple calculations were performed).

\begin{tabular}{lccc}
\hline \hline $\mathrm{E}_{\text {binding }}[\mathrm{eV}]$ & \multicolumn{3}{c}{ Anatase facet } \\
& $(100)$ & $(001)$ & $(101)$ \\
\hline $\mathrm{Pt}_{13}$ cubo $(111)$ & -3.87 & -14.58 & -3.71 \\
$\mathrm{Pt}_{55}$ cubo $(111)$ & -1.34 & -12.31 & -0.42 \\
$\mathrm{Pt}_{55}$ cubo $(100)$ & & & -0.29 \\
$\mathrm{Pt}_{55}$ ico $(111)$ & & & -2.32 \\
\hline \hline
\end{tabular}

The $\mathrm{Pt}_{55}$ cuboctahedral nanoparticle also binds strongly to the (001) facet, with $-2.05 \mathrm{eV}$ per interfacial Pt atom. Very similar surface reconstructions are observed, though the 
nanoparticle is less distorted due to its larger size. Again, this effect on the surface contributes greatly to the binding energy of the nanoparticle to the surface.

Comparing the two different sized nanoparticles, our calculations show that the larger, 55 atom nanoparticle binds to each anatase facet considerably less strongly (from $\sim 2.3 \mathrm{eV}$ to $\sim 3.3 \mathrm{eV}$ weaker for the full nanoparticle) than the 13 atom one. The smaller nanoparticle deforms much more than the larger one, which is due to the lower activation barrier for it to deform/reconstruct.

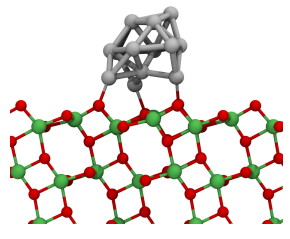

(a) (101)

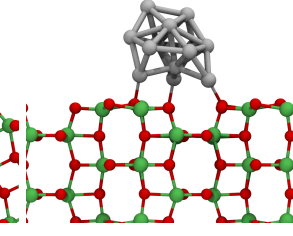

(b) (100)

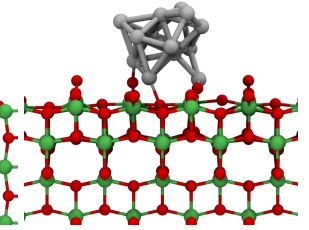

(c) (001)
FIG. 1. Geometry optimised $\mathrm{Pt}_{13}$ cuboctahedra on (a) (101), (b) (100) and (c) (001) anatase facets. O, Pt and Ti in red, silver and green, respectively.

While the binding strength of the $\mathrm{Pt}_{13}$ on anatase (101) and (100) surfaces is much weaker than the (001) surface, the interaction in all cases is strong enough to deform the nanoparticle substantially. The deformed structures, seen in Figure 1, seem to retain the 3 layers of the geometric nanoparticle, but the facets become difficult to define and there is significant wetting to the surface.

The $\mathrm{Pt}_{55}$ cuboctahedral nanoparticle, deposited via its (111) facet onto the anatase (101) suface, is deformed only slightly, with an expansion of $\sim 0.06 \AA$ in Pt-Pt bonds in the interfacial layer, and a contraction of $\sim 0.08 \AA$ of the interlayer bonds of the outermost and first subsurface layer. A greater level of deformation is observed when the same nanoparticle is deposited on its (100) facet. In terms of bond lengths, the opposite effect is observed: interfacial $\mathrm{Pt}-\mathrm{Pt}$ bonds remain largely unchanged, contracting at most by $0.02 \AA$, while the bonds between the central atom of interfacial layer and its neighbours above it increase by a much more significant $\sim 0.2 \AA$ as the atom is pulled toward the anatase surface, as shown in Figure 2(b). For either facet of the $\mathrm{Pt}_{55}$ cuboctahedron, the binding to anatase (101) is very weak: $-0.07 \mathrm{eV}$ per interfacial atom for the $\mathrm{Pt}_{55}$ (111) facet and $-0.03 \mathrm{eV}$ per interfacial atom for the $\mathrm{Pt}_{55}$ (100) facet).

With that said, the activation barrier for a Pt nanoparticle to reconstruct on a metal oxide surface is typically not that high, even for nanoparticles larger than those studied here ${ }^{43}$. This means that at finite temperature, such reconstructions, which could lower the free energy of the final system considerably (and thus increase the binding energy), might become very likely to occur. These reconstructions include wetting to the surface, shifts to and from various motifs (icosahedra, Marks decahedra, FCC crystals etc.) and deformation of facets, especially those in contact with the support, to reduce the total energy. It should also be noted that the smaller nanoparticles have a heightened sensitivity to the deposition site, while larger nanoparticles feel an averaged effect over many atoms.

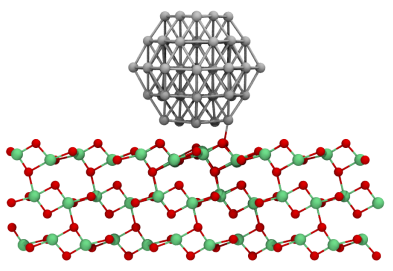

(a) cubo (111)

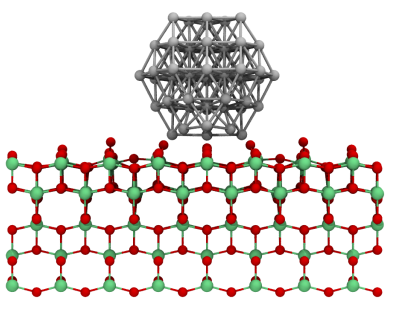

(c) cubo (111)

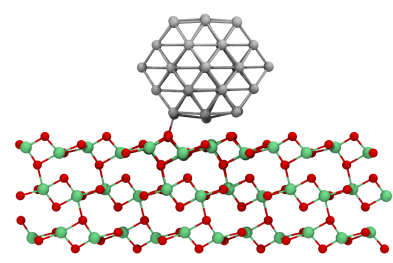

(b) cubo (100)

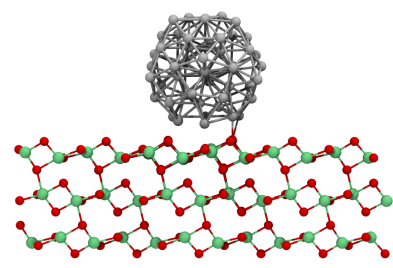

(d) ico
FIG. 2. Geometry optimised $\mathrm{Pt}_{55}$ cuboctahedra and icosahedron on (a, b, d) anatase (101) and (c) anatase (001). O, Pt and Ti in red, silver and green, respectively.

The icosahedral nanoparticle is deformed more than the cuboctahedra in either case. The interfacial Pt atoms shift in such a way as to sit atop surface oxygens where possible, increasing Pt-Pt bond lengths of interfacial atoms, and breaking them entirely in some cases. This causes Pt atoms in the above layers to shift down to maintain their bonds with interfacial atoms that separate from one another. The process could be described as a wetting to the surface, or at least the early stages of such a process, although there is still some activation barrier preventing this fully occurring at zero temperature.

TABLE III. Electron transfer between nanoparticle and support, calculated via Bader $^{44}$ and Mulliken analyses. Units in number of $\mathrm{e}^{-}$, with a negative value corresponding to a transfer of electrons from the nanoparticle to the anatase support.

\begin{tabular}{lccc}
\hline \hline Nanoparticle & Anatase facet & \multicolumn{2}{c}{$\mathrm{e}^{-}$transfer } \\
& & Bader & Mulliken \\
\hline $\mathrm{Pt}_{13}$ cubo (111) & $(100)$ & -0.51 & -0.25 \\
$\mathrm{Pt}_{13}$ cubo $(111)$ & $(001)$ & -0.56 & -0.35 \\
$\mathrm{Pt}_{13}$ cubo (111) & $(101)$ & -0.50 & -0.22 \\
$\mathrm{Pt}_{55}$ cubo $(111)$ & $(101)$ & -0.54 & -0.03 \\
$\mathrm{Pt}_{55}$ cubo $(100)$ & $(101)$ & -0.54 & 0.11 \\
\hline \hline
\end{tabular}

Charge transfer between the support and the deposited nanoparticle contributes to its binding strength, and also implies a shift in its electronic structure. With a Bader population analysis ${ }^{44}$, we observe a consistent transfer of around 0.5 electrons from the Pt nanoparticle to the anatase support, regardless of nanoparticle size or anatase facet. These results can be seen in Table III, along with a Mulliken analysis, which is consistent in direction of transfer (except in the case of $\mathrm{Pt}_{55}$ deposited on its (100) facet), but shows a smaller transfer in all cases. 


\section{B. Effects on Ligand Binding}

\section{Oxygen on $\mathrm{Pt}_{13}$}

The binding energies of oxygen to $\mathrm{Pt}_{13}$ in isolation and supported on each of the anatase facets are given in Table IV. The bridge sites are found to be the most favourable on isolated $\mathrm{Pt}_{13}$. This is in contrast to binding energies calculated with frozen nanoparticles ${ }^{27,45}$, where the hollow (HCP) site is favourable. The $\mathrm{Pt}_{13}$ nanoparticle undergoes a significant shift in Pt-Pt bond lengths and structure upon oxygen deposition on the atop and especially bridge sites, in a way that is not seen with the hollow site.

TABLE IV. Binding energies of oxygen (using $0.5 \mathrm{O}_{2}$ as a reference) on $\mathrm{Pt}_{13}$ on different anatase surfaces. Although significant distortion of the $\mathrm{Pt}_{13}$ nanoparticles occurs upon deposition onto the anatase support, making it impossible to define low index facets for the nanoparticles themselves, it is still possible to assign atop, bridge, and hollow sites. For a nanoparticle of this size, all bridge sites are essentially edges, while top sites are vertices. There are no (100)like facets on these nanoparticles after deposition on anatase, and oxygens on hollow sites have a coordination of 3 (rather than possibly 4$).{ }^{*}$ For the nanoparticles on the anatase (101) and (001) facets, the hollow site was not stable, and minimised to a bridge site instead.

\begin{tabular}{ccccc}
\hline \hline & & \multicolumn{3}{c}{$\mathrm{Pt}_{13}+$ Anatase support } \\
{$[\mathrm{eV}]$} & Isolated $\mathrm{Pt}_{13}$ & $(100)$ & $(001)$ & $(101)$ \\
\hline $\mathrm{E}_{\text {atop }}$ & -2.80 & -1.83 & -1.90 & -1.58 \\
$\mathrm{E}_{\text {bridge }}$ & -4.88 & -1.83 & -1.17 & -1.10 \\
$\mathrm{E}_{\text {hollow }}$ & -2.04 & -0.99 & $-1.10^{*}$ & $-1.61^{*}$ \\
\hline \hline
\end{tabular}

Given the significant changes to the nanoparticle structures on the anatase facets, comparing sites like for like is not possible. However, some trends can still be established. There is a weakening of the binding strength of oxygen on titania supported nanoparticles of this size, irrespective of the titania facet they are deposited on. The strongest binding site of oxygen on a supported nanoparticle is an atop site on a (001) anatase facet (Figure 3c), and is $\sim 3 \mathrm{eV}$ weaker than the most favourable site overall on the unsupported nanoparticle, and $0.9 \mathrm{eV}$ weaker than its most favourable atop site. The most favourable binding site on the $\mathrm{Pt}_{13}$ nanoparticle for each anatase facet are shown in Figure 3.

It must be noted that the hollow sites on the (001) and (101) supported nanoparticles (the latter of which is shown in Figure 3a) that were tested do not appear to be stable, relaxing instead to a bridge site. A different bridge site on the (101) supported nanoparticle binds oxygen $0.5 \mathrm{eV}$ less strongly than this, while for (001) another bridge site binds almost $0.3 \mathrm{eV}$ more strongly. This seems to indicate that the high levels of disorder in these small nanoparticles have a greater effect than the coordination of the bound oxygen. The strongest binding site of any supported nanoparticle was the top site of the anatase (001) supported nanoparticle. Given the high level of variation in binding between sites of the same type (atop, bridge, hollow), and further variation arising from choosing a different deposition site for the nanoparticle, we can only con-

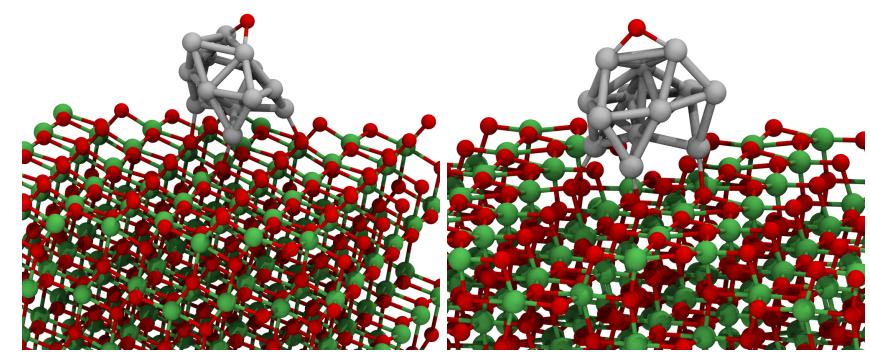

(a) (101) hollow*

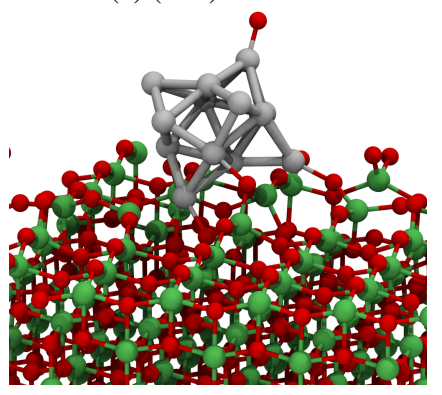

(b) (100) bridge

(c) (001) atop

FIG. 3. Most favourable binding positions following geometry relaxation of $\mathrm{O}$ on $\mathrm{Pt}_{13}$ deposited on the (a) (101), (b) (100) and (c) (001) anatase surface. In the case of (a), the hollow site relaxed to a bridge site (as did all tested hollow sites). For (b), the atop and bridge sites have the same energy, though only the bridge site is shown. $\mathrm{O}, \mathrm{Pt}$ and $\mathrm{Ti}$ in red, silver and green, respectively.

clude that the support weakens the binding energy of oxygen, rather than drawing more detailed conclusions about specific sites.

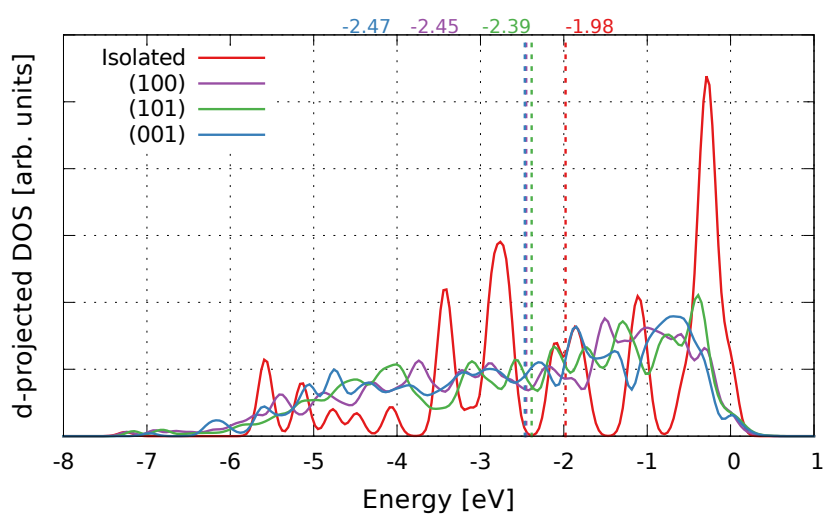

FIG. 4. d-band plots for the $\mathrm{Pt}_{13}$ cuboctahedra in isolation and on anatase (100), (001) and (101), projected to all the Pt atoms. Vertical dashed lines show the d-band centre, with the corresponding values (in $\mathrm{eV}$ ) given above. Energy is relative to the calculated Fermi energy.

It is interesting to explore if various electronic descriptors, which typically are expected to help rationalise and even predict binding energies without explicit calculations, perform in this case where the nanoparticle is heavily deformed due to the interaction with the support. The d-projected density of states, 
normally localised to atoms at the surface of a metal, has often been used to predict the properties of that surface. In particular, the d-band model ${ }^{46,47}$, which includes the d-band centre and d-band width ${ }^{38}$ as descriptors, has been used over the last 2 decades for a variety of systems ${ }^{48}$, including Pt surfaces. The d-band centre can be used to predict binding energies of $\mathrm{O}$ and other ligands on Pt surfaces as low values of the d-band centre correlate with low binding energies and vice versa.

Because the $\mathrm{Pt}_{13}$ nanoparticles are heavily deformed upon deposition, it is not possible to assign facets to their surface. Therefore, for this nanoparticle, we chose to compute density of states projected onto the d-bands for all the Pt atoms (i.e. the full nanoparticle). The obtained densities of states are shown in Figure 4 and as we can see, there is a dramatic drop of the d-band centre from the isolated $\mathrm{Pt}_{13}$, which has a value of $-1.98 \mathrm{eV}$, to the cases where it is adsorbed on the titania (100), (101), and (001) surfaces with values $-2.45 \mathrm{eV}$, $-2.39 \mathrm{eV}$, and $-2.47 \mathrm{eV}$, respectively. This drop correlates with the $\mathrm{O}$ adsorption energies following the expected weakening of $\mathrm{O}$ adsorption with decreasing d-band centre value. However, the ordering between the different supported nanoparticles is reversed, with strongest oxygen binding site on the anatase (001) supported nanoparticle being almost $0.3 \mathrm{eV}$ stronger than that on the anatase (101) facet, while having a $\mathrm{d}$-band centre $0.08 \mathrm{eV}$ lower. Once again, the geometry of the nanoparticles strongly affects the binding strength, and the dband centre values are very similar for all supported systems.

What is also clear from the isolated $\mathrm{Pt}_{13}$ PDOS plot is that there is no well defined "band" with only 13 atoms, and the electronic structure more closely resembles a combination of perturbed atomic orbitals than the metallic bands of extended Pt systems. Nevertheless, this descriptor can still serve the purpose of predicting the effect of a support on $\mathrm{O}$ binding. We also note the interesting observation that the $\mathrm{Pt}_{13}$ nanoparticle appears to become more metallic when supported, with a more smeared out band structure than is present for the isolated case. We also performed a DOS analysis on the local anatase surface directly beneath the nanoparticle, to see whether this also becomes more metallic. The results are given in Figure 2 of the supplementary material. In summary, we do observe an increase in the DOS in the band gap region, with non-zero DOS much closer to the Fermi level than for the slab alone. Most of this contribution seems to come from the oxygen $\mathrm{p}$ states. Without full conduction band calculations, however, we cannot draw further conclusions about the band gap of the $\mathrm{TiO}_{2}$ with a nanoparticle supported on it.

The charge transfer (Table III) and d-band centre ordering for $\mathrm{Pt}_{13}$ on the different anatase facets is the consistent, with the anatase (001) drawing the most electron density from the nanoparticle and also having the lowest d-band centre, followed by the (100) and then (101) facet. As electrons near the Fermi level are the most likely to transfer away from the nanoparticle (they are in the highest occupied energy states and therefore the most weakly bound), it seems sensible that an electron depletion would cause a downward shift in the dband centre.

Another electronic descriptor, based on the electron density at an isosurface of the electrostatic potential and developed in previous work $^{28}$, can aid prediction of oxygen binding energy on Pt nanoparticles. In that work, we demonstrated a useful correlation between oxygen binding energy and electron density, provided atop, bridge and hollow sites are considered separately. Here, we have calculated this descriptor for each site on the isolated and supported nanoparticles and compared them with the explicit binding energies. The results are shown in Figure 3 of the supplementary material. For these $\mathrm{Pt}_{13}$ systems, there appears to be no obvious correlation between the binding energy and the electronic descriptor, which we believe is due to the small size and distorted shapes of these nanoparticles.

Overall, for the $\mathrm{Pt}_{13}$ nanoparticle, its small size and tendency to reconstruct upon deposition or even due to the binding of oxygen (on the bridge site especially) means that electronic effects cannot be studied in isolation from geometric effects, so electronic descriptors are of limited use for such small nanoparticles.

\section{Oxygen on $\mathrm{Pt}_{55}$}

We are able to more directly compare binding sites on $\mathrm{Pt}$ facets on the larger $\mathrm{Pt}_{55}$ cuboctahedon, as it is deformed far less than $\mathrm{Pt}_{13}$ upon deposition. For the isolated nanoparticle, there are 9 unique binding sites, as shown in Figure 5. While we refer to the distinct facets of the cuboctahedral nanoparticle as the (111) (triangular) and (100) (square) for the sake of convenience, it is important to note that their properties, including their geometries, are quite distinct from the extended slabs from which they take their names ${ }^{49}$. The finite size effects modify the lattice spacing, electronic structure and remove symmetries compared to an extended slab.

Table $\mathrm{V}$ shows the oxygen binding energies to the $\mathrm{Pt}_{55}$ nanoparticle. In the case of the isolated nanoparticle, we see that the bridge (edge) site is the strongest binding at -1.59 $\mathrm{eV}$, followed by a clustering of very similarly binding strength sites, in the range -1.30 to $-1.42 \mathrm{eV}$, including the atop (vertex), hcp hollow and the (100) and (111) bridge sites (which in fact relaxed to hcp hollow sites). Distinctly lower in binding energy are the fcc hollow site, the atop (edge), and atop (100), which have binding energy $-0.91,-0.73$ and $-0.59 \mathrm{eV}$, respectively.

Unlike for $\mathrm{Pt}_{13}$, our results, which have been obtained with full relaxation of the isolated nanoparticle-O system, are much more in line (same energy ordering and similar absolute binding energy values) with the frozen isolated nanoparticle calculations in the literature 27,45 . Again, the larger nanoparticle's tendency not to deform nearly as much upon oxygen adsorption is the reason for this observation.

$\mathrm{O}$ binding to supported $\mathrm{Pt}_{55}$ shows the support effects are already less significant for the larger (but still only $\sim 1 \mathrm{~nm}$ in diameter) nanoparticle. While the bridge, hollow and one of the atop sites follow the same trend of the support weakening the binding strength, the atop vertex and (100) sites show an increase of 0.05 and $0.13 \mathrm{eV}$, respectively. While the former $\sim 3.5 \%$ increase is small, the latter, at $\sim 22 \%$, is significant. It is not immediately clear as to why this increase is observed, 


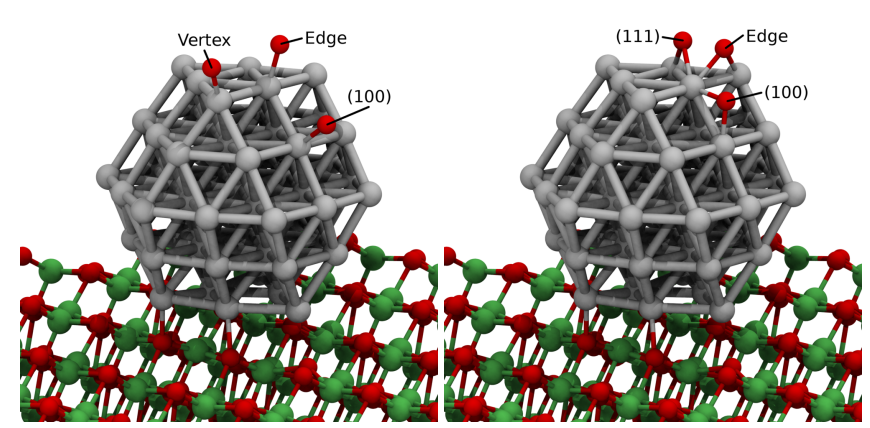

(a) atop sites

(b) bridge sites

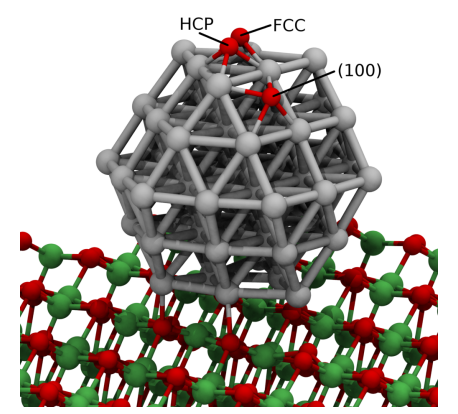

(c) hollow sites

FIG. 5. The (a) atop, (b) bridge and (c) hollow binding sites calculated on the $\mathrm{Pt}_{55}$ cuboctahedron, shown here with oxygen and on the anatase (101) support to illustrate where the binding sites we have calculated are (relative to the support). For the isolated nanoparticle, these are all of the unique sites. Each binding energy calculation was performed separately, they are combined here for illustrative purposes only.

TABLE V. Binding energies of oxygen (using half of the energy of isolated $\mathrm{O}_{2}$ as a reference) to $\mathrm{Pt}_{55}$ cuboctahedral on the anatase (101) surface. All 9 unique sites on an isolated $\mathrm{Pt}_{55}$ nanoparticle are calculated. The most favourable binding site (boldface) is the bridge edge site in both isolated and supported cases. ${ }^{*}$ The (111) bridge site relaxed to HCP hollow in both isolated and supported cases.

\begin{tabular}{lccc}
\hline \hline [eV] & ${\text { Isolated } \mathrm{Pt}_{55}}$ & $(101)$ & Frozen \\
\hline $\mathrm{E}_{\text {atop }}^{\text {edge }}$ & -0.73 & -0.55 & -0.40 \\
$\mathrm{E}_{\text {attop }}^{\text {verto }}$ & -1.42 & -1.47 & -1.37 \\
$\mathrm{E}_{\text {atop }}^{(100)}$ & -0.59 & -0.72 & 0.06 \\
$\mathrm{E}_{\text {bridge }}^{\text {edge }}$ & $\mathbf{- 1 . 5 9}$ & $\mathbf{- 1 . 5 0}$ & -1.24 \\
$\mathrm{E}_{\text {bridge }}^{(111)}$ & $-1.36^{*}$ & $-1.28^{*}$ & $-1.03^{*}$ \\
$\mathrm{E}_{\text {bridge }}^{(100)}$ & -1.42 & -1.30 & -0.99 \\
$\mathrm{E}_{\text {hollow }}^{\text {hhp }}$ & -1.35 & -1.28 & -1.05 \\
$\mathrm{E}_{\text {hollow }}^{\text {fcc }}$ & -0.91 & -0.87 & -0.70 \\
$\mathrm{E}_{\text {hollow }}^{(100)}$ & -1.32 & -1.26 & -1.03 \\
\hline \hline
\end{tabular}

and there are many factors to consider. The atop vertex site is the lowest coordinated (5 first neighbours) of any site, while the (100) atop site is on the central (100) Pt atom, which has 8 first neighbours. For reference, the atop edge site has 7 first neighbours. For the isolated system, the binding energy ordering follows the ordering of neighbours, but this is not true for the supported system, where the edge and (100) sites switch order. However, these two sites, as for the isolated case, are the weakest $\mathrm{O}$ binding sites, at $-0.55 \mathrm{eV}$ for the edge site and $-0.72 \mathrm{eV}$ for the (100) site, which makes them less likely to play a role in a chemical process involving oxygen adsorption, as all other sites are preferred. These are in fact the only sites where the energy ordering differs between the isolated and supported systems.

In an attempt to distinguish the contribution to the $\mathrm{O}$ binding energies of the geometric and electronic effects of the support, we performed binding energy calculations using an isolated $\mathrm{Pt}_{55}$ nanoparticle, frozen with the geometry it had after deposition on anatase (101). The results are given in the rightmost column of Table V. While this is obviously not a physically realistic calculation, nor a rigorous method of decomposition of energies, it can nevertheless provide some interesting insight. With the exception of the atop (100) site, it would appear as though the geometric effects are the dominant ones, accounting for at least $70 \%$ of the binding energy. The electronic effects are still significant, however, and do not affect the sites in a uniform way. The bridge edge site, which is the strongest binding site in the isolated and supported cases, is not as strongly binding as the atop vertex site for the frozen nanoparticle, indicating that the electronic effects are more significant for the bridge site. In general, it seems as though the geometric effects weaken the oxygen binding, while the electronic effects partially counteract this, strengthening the binding energy by a smaller amount.

We can use the d-band model again for $\mathrm{Pt}_{55}$ to assess the electronic effects. For this larger nanoparticle, the cuboctahedral (100) and (111) facets remain well defined on the anatase surfaces, and the PDOS can be evaluated for each individual surface.

The two facets on the isolated system share all but one atom. Every atom on the (111) facet is at an edge or vertex position, which are also edges and vertices of the (100) facet. The central atom on the (100) facet is the only atom not shared by the two facets. It is therefore unsurprising that the PDOS d-band projections, shown in Figure 6, are so similar in shape (once scaled to the total number of electrons/atoms projected onto: 9:6 in this case). Both facets also behave similarly upon deposition, with a downward shift in the d-band centre of 0.24 $\mathrm{eV}$ and $0.19 \mathrm{eV}$ for the (111) and (100) facets, respectively. As per the d-band model, this should result in a decrease in the binding energy of oxygen (and other adsorbates), which is what we observe for most sites. We should perhaps also observe the binding energies on the (111) facets to decrease slightly more than on the (100), and this does appear to be the case for the hollow sites (though by only $0.01 \mathrm{eV}$ ), but not for bridge sites. There are no comparable atop sites since there are no (111) atop sites on $\mathrm{Pt}_{55}$ that are not edge or vertex sites. We are unable to use the d-band model here to elucidate why the two atop (vertex and (100)) sites increase in binding energy when supported, as the model is averaged over entire facets, and is anyway less suited for low coordination sites.

Comparing again with the charge transfer of 0.54 electrons (using Bader partitioning, from Table III) from the nanoparticle to anatase support, see the same trend as before: a de- 


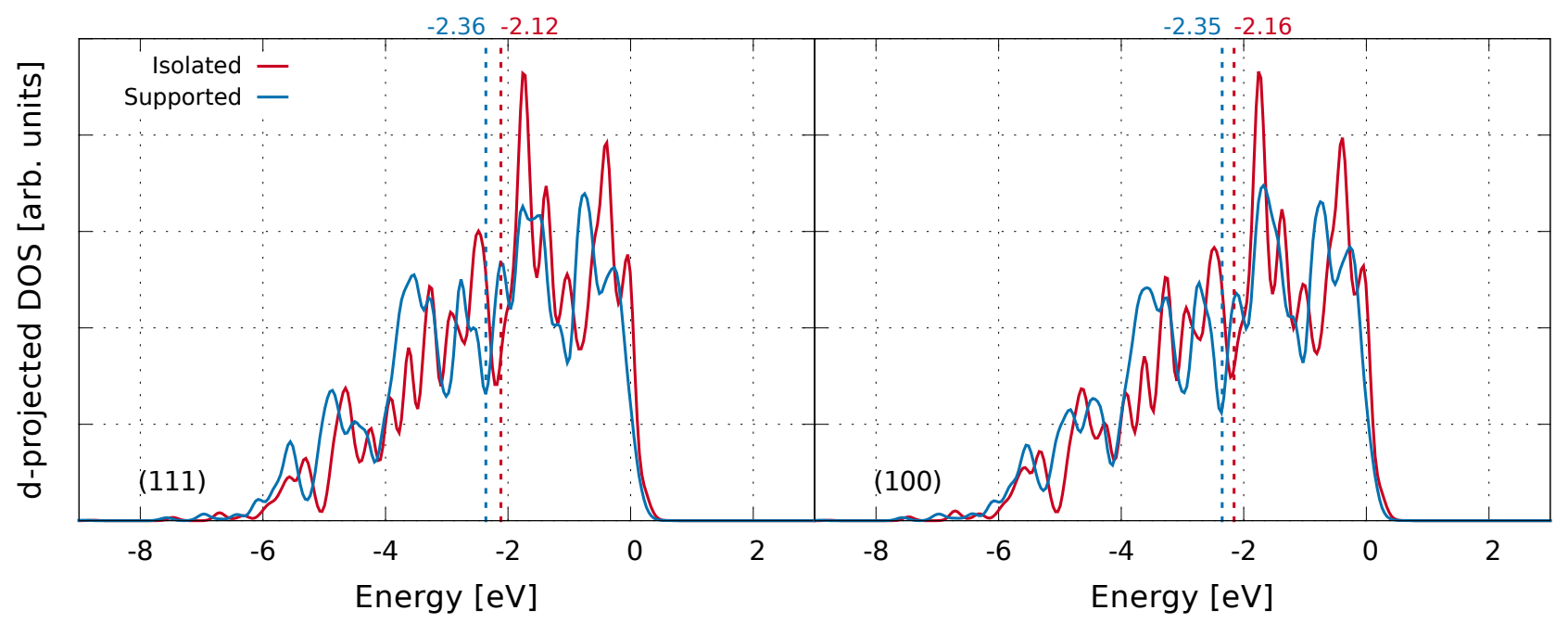

FIG. 6. d-band DOS plots for the topmost (111) and (100) facets of the $\mathrm{Pt}_{55}$ cuboctahedra on anatase (101). Vertical dashed lines show the $\mathrm{d}$-band centre (values in $\mathrm{eV}$ ). Energy is relative to the calculated Fermi level.

pletion of electrons coincides with a lowering of the d-band centre. It is likely that the charge transfer we observe contributes significantly to the d-band centre shift.

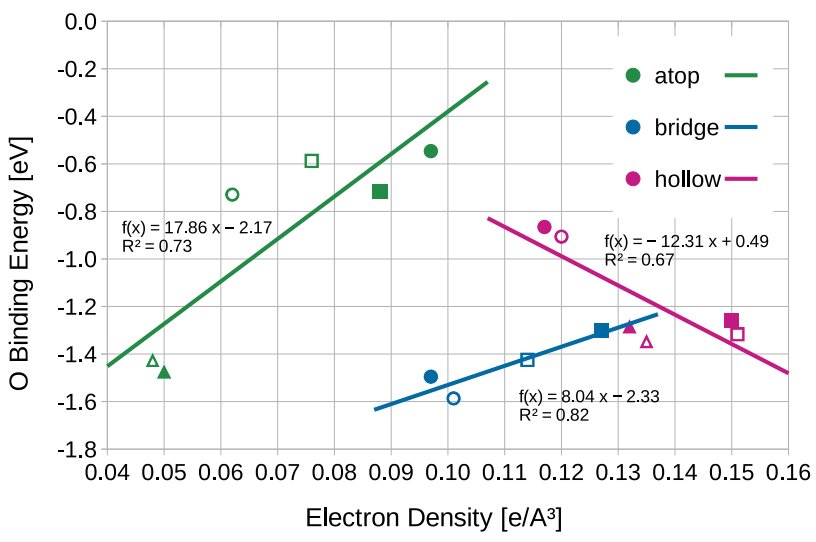

FIG. 7. Calculated oxygen binding energies against the electron density descriptor for all unique atop, bridge and hollow sites on an isolated (hollow) $\mathrm{Pt}_{55}$ nanoparticle, as well as the same sites (on the nanoparticle hemisphere away from the interface) after deposition on anatase (101) (filled). Each site within each group (atop, bridge, and hollow) is distinguished by its own shape. A square signifies the (100) site of each group, while the atop vertex and hcp hollow sites are triangles. Finally, the atop edge, (111) bridge and fcc hollow sites are circles.

Figure 7 shows a moderate correlation between the electronic descriptor and the oxygen binding energy, again with a separation of different site types. Unlike in our previous work, the hollow sites show a reversed trend, where increases in electron density on the isosurface implies a stronger binding. Since the descriptor uses the position of an adsorbate as initialised for a binding energy calculation, rather than a relaxed geometry (since we want to use this descriptor to avoid explicit binding energy calculations), it performs poorly when the ligand relaxes to a different site. This is the case for the (111) bridge site (supported and unsupported), which have been excluded from the plot. The sites they relax to, (hcp hollow), are in fact very close to the bridge site trend line (the two purple points at 0.132 and $0.135 \mathrm{e}^{-} \AA^{-3}$ ).

The descriptor is also sensitive to the effects of the support, and points are not clustered by supported and isolated systems. While we see a consistent shift (with the overall trend line) of binding energy and descriptor with the hollow sites, this is not the case for two of the atop and one of the bridge sites. For the two atop sites, this is because the calculated binding energies tend This means the descriptor could be a useful tool in assessing the suitability of these supports as catalysts for a given application.

\section{Carbon Monoxide on $\mathrm{Pt}_{55}$}

Carbon monoxide is another important ligand to consider when assessing nanoparticle catalysts, as it is directly involved in many industrial chemical processes, such steam reforming and the water-gas shift reaction, as well as being present in automotive exhaust gases. It is also useful as a probe molecule, as carbon monoxide binding energy tends to correlate strongly with the binding energies of larger hydrocarbons and other, more complex molecules. Here, we have performed the same binding energy calculations for $\mathrm{CO}$ as we did for oxygen on $\mathrm{Pt}_{55}$ on the anatase (101) surface.

There are well known issues with DFT calculations of CO binding to the Pt (111) surface slabs ${ }^{50}$. Specifically, DFT calculations tend to overestimate the preference for hollow sites over atop sites, in contrast to experimental results. Our results on $\mathrm{Pt}_{55}$ nanoparticles, presented in Table VI do not show this behaviour, with the atop sites being preferred. This is because the undercoordination of Pt atoms on edges and vertices, which are not present on extended slabs, strengthens the binding of $\mathrm{CO}$ enough to reverse the order back to favouring 
TABLE VI. Binding energies of carbon monoxide to $\mathrm{Pt}_{55}$ cubo on the anatase (101) surface. ${ }^{*}$ The (111) bridge site relaxed to HCP hollow in both isolated and supported cases.

\begin{tabular}{lcc}
\hline \hline$[\mathrm{eV}]$ & ${\text { Isolated } \mathrm{Pt}_{55}}$ & $(101)$ \\
\hline $\mathrm{E}_{\text {atop }}^{\text {edge }}$ & -2.03 & -1.89 \\
$\mathrm{E}_{\text {atortex }}^{\text {verto }}$ & $\mathbf{- 2 . 1 2}$ & -1.97 \\
$\mathrm{E}_{\text {atop }}^{(100)}$ & -1.92 & -1.85 \\
$\mathrm{E}_{\text {bridge }}^{\text {edge }}$ & -1.89 & -1.82 \\
$\mathrm{E}_{\text {bridge }}^{(111)}$ & $-1.72^{*}$ & $-1.64^{*}$ \\
$\mathrm{E}_{\text {bridge }}^{(100)}$ & $\mathbf{- 2 . 1 2}$ & $\mathbf{- 2 . 0 6}$ \\
$\mathrm{E}_{\text {hollow }}^{\text {hcp }}$ & -1.72 & -1.65 \\
$\mathrm{E}_{\text {hollow }}^{\text {fcc }}$ & -1.15 & -1.09 \\
$\mathrm{E}_{\text {hollow }}^{(100)}$ & -1.65 & -1.53 \\
\hline \hline
\end{tabular}

atop sites ${ }^{27}$. As stated previously, for $\mathrm{Pt}_{55}$, all 6 of the atoms on each (111) facet belong either to an edge or a vertex. The underlying issue of DFT and CO binding on hollow vs top sites still remains, however, and should be considered when analysing these results.

For isolated $\mathrm{Pt}_{55}$, the (100) bridge and atop vertex sites are the most favourable, both at $-2.12 \mathrm{eV}$, followed by the atop edge site at $-2.03 \mathrm{eV}$. The addition of the support reduces the binding strength in all cases, with the atop vertex and edge sites seeing the largest decreases $(0.15 \mathrm{eV}$ and $0.13 \mathrm{eV})$, and the (100) hollow site almost the same with a $0.12 \mathrm{eV}$ decrease. All other sites decreased fairly uniformly, by between 0.06 and $0.08 \mathrm{eV}$. In fact, the energy ordering (site preference) of all sites is unchanged by deposition onto anatase (101). The relaxed geometry of the supported nanoparticle with $\mathrm{CO}$ on the most favourable atop, bridge and hollow site are shown in Figure 8.

DFT calculations on extended surfaces ${ }^{51}$ suggest that the $\mathrm{Pt}$ (111) surface binding sites all bind $\mathrm{CO}$ at around $-2.0 \mathrm{eV}$, with a small spread of about $\pm 0.1 \mathrm{eV}$, with the fcc hollow site falling in the middle between hcp hollow (strongest binding) and atop (weakest binding). In our case, the fcc site binds much less strongly than the other (111) sites, and $\sim 0.56$ $\mathrm{eV}$ weaker than the hcp site in particular (both for supported and unsupported nanoparticles). By contrast, on a slab, it is weaker by only $0.09 \mathrm{eV}$. Once again, the geometry of the nanoparticle can explain this result: the fcc site is at the centre of the $\mathrm{Pt}_{55}$ (111) facet, so it is less affected by the undercoordination of $\mathrm{Pt}$ atoms at the edges and vertices.

Following from this, we see a much larger range of binding energies for $\mathrm{CO}$ on different sites compared to extended slabs, which should mean a lower rate of $\mathrm{CO}$ diffusion as the free energy change of moving site increases. The range of $\mathrm{CO}$ binding energies on the $\mathrm{Pt}_{55}$ (111) facet is $0.97 \mathrm{eV}$ for the isolated nanoparticle, and $0.88 \mathrm{eV}$ when supported, compared to just $0.22 \mathrm{eV}$ on an extended (111) surface.

If we take (approximate) the migration energy barrier to be the range of site binding energies, then, at room temperature $\left(\mathrm{k}_{b} \mathrm{~T}\right.$ of $\left.0.026 \mathrm{eV}\right)$, we would expect $\mathrm{CO}$ diffusion to occur on an extended slab, and to do so via hopping events from

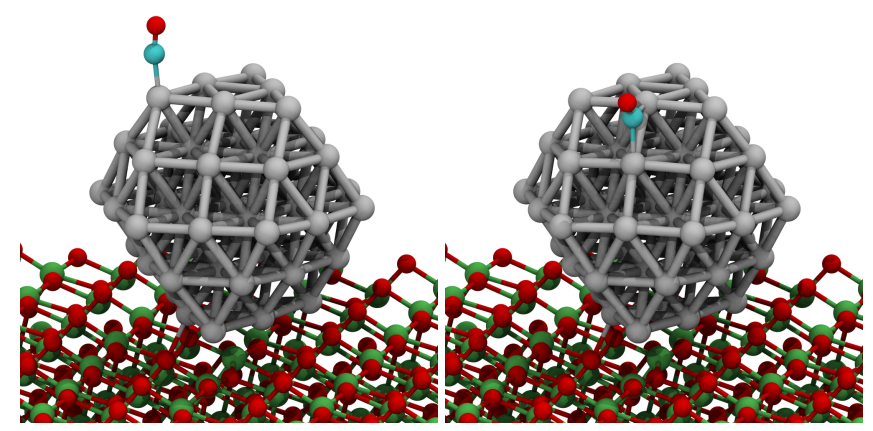

(a) atop vertex

(b) (100) bridge

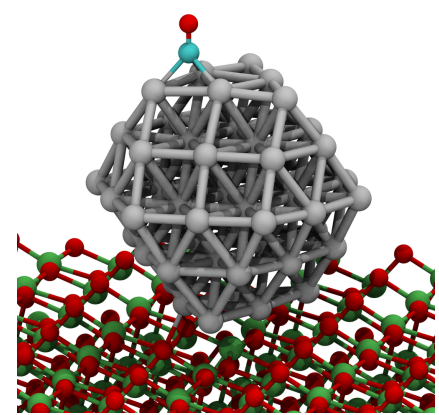

(c) HCP hollow

FIG. 8. Optimised binding positions of $\mathrm{CO}$ on $\mathrm{Pt}_{55}$ cuboctahedra on anatase (101). The most favourable (a) atop, (b) bridge and (c) hollow binding sites are shown. O, C, Pt and Ti in red, light blue, silver and green, respectively.

one adsorption site to another ${ }^{52}$. The much higher range on the $\mathrm{Pt}_{55}$ nanoparticle means this barrier is likely to be much higher. The minor reduction in range upon deposition of the nanoparticle suggests the support effects could improve CO diffusion on the surface, although it still very far from the extended slab. Also, if we consider the whole nanoparticle, including the (100) facet, the range is actually unchanged by the support, due to the relatively small downward shift of the (100) bridge site compared to the atop vertex site.

Considering $\mathrm{CO}$ binding energies against our electronic descriptor in Figure 9 shows a reasonable correlation for atop sites, a weak but similarly (to oxygen) reversed hollow site correlation, and a weak reversed correlation (compared to $\mathrm{O}$ ) for bridge sites. While the atop and hollow sites shift in binding energy and descriptor in a consistent way upon deposition (broadly shifting along in the direction of the trend line), this does not happen with the bridge sites, where the (100) site increases in electron density as the binding energy weakens.

Although the binding mechanisms to metal surfaces are different for $\mathrm{O}$ and $\mathrm{CO}$, it is often found that the binding strength of one correlates strongly with that of the other across a wide variety of metal systems ${ }^{53}$. It is therefore particularly pleasing that we see a similar correlation for $\mathrm{CO}$ as we did for $\mathrm{O}$ with the electronic descriptor. While in the case of $\mathrm{CO}$, the descriptor does not appear to be applicable to predict bridge site binding, it shows good correlation for top and hollow sites and could be used to provide predictive insights for such sites. Ultimately, this descriptor is intended as a way of screening 


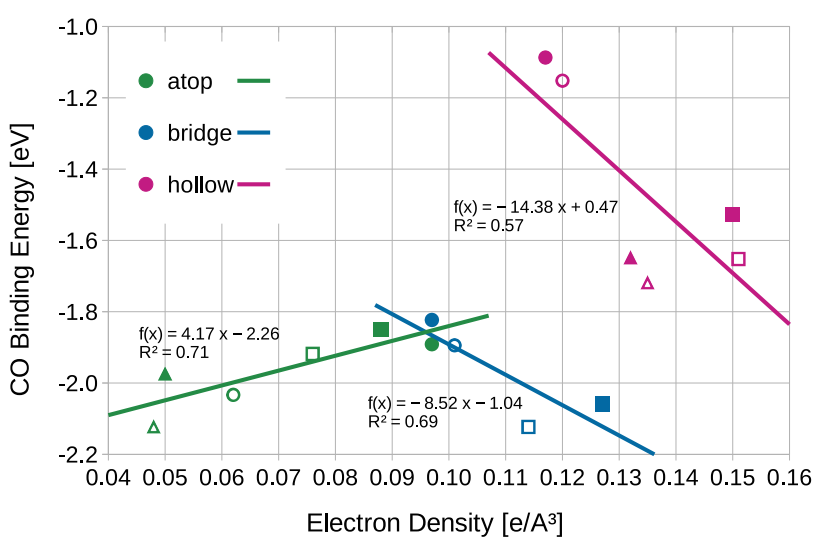

FIG. 9. Calculated CO binding energies against the electron density descriptor for all unique atop, bridge and hollow sites on an isolated (hollow) $\mathrm{Pt}_{55}$ nanoparticle, as well as the same sites (on the nanoparticle hemisphere away from the interface) after deposition on anatase (101) (filled). Each site within each group (atop, bridge, and hollow) is distinguished by its own shape. A square signifies the (100) site of each group, while the atop vertex and hcp hollow sites are triangles. Finally, the atop edge, (111) bridge and fcc hollow sites are circles.

potential nanoparticle catalyst systems at a higher throughput than would be possible with explicit binding energy calculations, so the somewhat low $\mathrm{R}^{2}$ values we see, particularly for the hollow sites with $\mathrm{CO}$, are still sufficient for this purpose.

\section{CONCLUSIONS}

We have carried out a computational study of the effects of an anatase support on the binding energies of oxygen and carbon monoxide on Pt nanoparticles. First of all, as far as the adsorption of Pt nanoparticles to anatase is concerned, the (001) facet of anatase is much stronger compared to the other facets, and due to its presence in the Wulff construction of anatase, it is likely that nanoparticles, at least of this size, would tend to deposit or anchor on this facet.

For the binding of small molecules to Pt nanoparticles, we find that the introduction of an anatase support leads to a weakening of $\mathrm{O}$ and $\mathrm{CO}$ binding energies. The nanoparticles we have modelled are small at $\sim 1 \mathrm{~nm}$ for $\mathrm{Pt}_{55}$, and it might be expected that larger nanoparticles would be less affected by the support, given that $\mathrm{Pt}_{55}$ is already affected much less than $\mathrm{Pt}_{13}$. We also find that oxygen binds much more strongly to isolated $\mathrm{Pt}_{13}$ than isolated $\mathrm{Pt}_{55}$, in part due to the deformation a single $\mathrm{O}$ can cause it to undergo, and while an anatase support reduces this binding strength by as much as $3 \mathrm{eV}$ (comparing the strongest binding sites in each case), supported $\mathrm{Pt}_{13}$ still has stronger oxygen binding sites than supported $\mathrm{Pt}_{55}$. We see a reduction in oxygen binding of $\sim 0.1 \mathrm{eV}$ on $\mathrm{Pt}_{55}$, which is a small but potentially useful amount, demonstrating how catalysts might be tuned via the use of this support to increase activity. If, for a given reaction, the catalytic activity is expected to be increased by a lower oxygen binding energy than unsupported (or weakly supported on something like graphene ${ }^{54}$ ) platinum, then an anatase support may well be a good choice of support, as binding energy is lowered on every low index facet tested here. Very similar decreases are seen with carbon monoxide binding, with the anatase support leading to lower binding energies overall. The behaviour is more consistent with $\mathrm{CO}$, with nearly all sites affected in the same way and by the same amount, and overall site preference remaining unchanged.

We have explored the use of electronic descriptors such as d-band centre and electron density-based descriptors for both isolated and supported Pt nanoparticles. For supported nanoparticles, the descriptors can provide semi-quantitative insights into how the binding is affected by the support, and therefore they could potentially be used to more quickly assess which supports (or support facets) might be suitable for a given catalytic process.

The d-band model is clearly sensitive to the introduction of a support, and the behaviour is consistent with existing literature, and could be useful even for small nanoparticles where the electronic structure is not fully metallic. Our electronic density and electrostatic potential based descriptor is also sensitive to the support effects, and we see a reasonable correlation between it and explicitly calculated oxygen binding energies, as long as the different sites are considered separately. The descriptor is also applicable to carbon monoxide, though fails to predict bridge site binding in a convincing manner, and the correlation is also somewhat low for hollow sites.

Interestingly, we see a consistent charge transfer of $\sim 0.5$ electrons from Pt nanoparticles to the anatase support, regardless of nanoparticle size or anatase facet. The $\mathrm{Pt}_{55}$ nanoparticle therefore has a lower electron depletion per atom $(\sim 4 \mathrm{x}$ lower) than the $\mathrm{Pt}_{13}$ one, and a weaker $\mathrm{O}$ and $\mathrm{CO}$ binding strength as well. Further work could perhaps explore this apparent correlation in more depth.

We also find that, at least for such small nanoparticles, it can be very difficult to separate geometric and electronic effects, and clearly both contribute significantly to the overall binding strength of ligands.

Our work helps to elucidate the complex interactions between metallic nanoparticles and metal oxide supports and provides useful insights for the design and tuning of heterogeneous catalysts.

\section{ACKNOWLEDGMENTS}

The authors acknowledge the use of the IRIDIS High Performance Computing Facility, and associated support services at the University of Southampton, in the completion of this work. We are grateful for computational support from the UK Materials and Molecular Modelling Hub, which is partially funded by EPSRC (EP/P020194), and the UK national high performance computing service, ARCHER. Access to both was obtained via the UKCP consortium and funded by EPSRC grant ref EP/P022561/1. TE would also like to thank Johnson Matthey and the EPSRC for financial support in the form of a Ph.D. studentship. 
${ }^{1}$ J. Seth, P. Dubey, V. R. Chaudhari, and B. L. V. Prasad, "Preparation of metal oxide supported catalysts and their utilization for understanding the effect of a support on the catalytic activity," New J. Chem. 42, 402-410 (2017).

${ }^{2}$ M. M. Schubert, V. Plzak, J. Garche, and R. J. Behm, "Activity, Selectivity, and Long-Term Stability of Different Metal Oxide Supported Gold Catalysts for the Preferential CO Oxidation in H2-Rich Gas," Catalysis Letters 76, 143-150 (2001).

${ }^{3}$ D. A. Peña, B. S. Uphade, and P. G. Smirniotis, "TiO2-supported metal oxide catalysts for low-temperature selective catalytic reduction of NO with NH3: I. Evaluation and characterization of first row transition metals," Journal of Catalysis 221, 421-431 (2004).

${ }^{4}$ E. Iglesia, "Design, synthesis, and use of cobalt-based Fischer-Tropsch synthesis catalysts," Applied Catalysis A: General 161, 59-78 (1997).

${ }^{5}$ S. Aranifard, S. C. Ammal, and A. Heyden, "On the importance of metal-oxide interface sites for the water-gas shift reaction over $\mathrm{Pt} / \mathrm{CeO} 2$ catalysts," Journal of Catalysis 309, 314-324 (2014).

${ }^{6}$ H. J. Park, S. H. Park, J. M. Sohn, J. Park, J.-K. Jeon, S.-S. Kim, and Y.K. Park, "Steam reforming of biomass gasification tar using benzene as a model compound over various Ni supported metal oxide catalysts," Bioresource Technology Supplement Issue on Recent Developments of Biomass Conversion Technologies, 101, S101-S103 (2010).

${ }^{7}$ M. Haruta, "Catalysis of Gold Nanoparticles Deposited on Metal Oxides," CATTECH 6, 102-115 (2002).

${ }^{8}$ E. A. Walker, D. Mitchell, G. A. Terejanu, and A. Heyden, "Identifying Active Sites of the Water-Gas Shift Reaction over Titania Supported Platinum Catalysts under Uncertainty," ACS Catal. 8, 3990-3998 (2018).

${ }^{9}$ R. Zennaro, M. Tagliabue, and C. H. Bartholomew, "Kinetics of Fischer-Tropsch synthesis on titania-supported cobalt," Catalysis Today 58, 309-319 (2000).

${ }^{10}$ A. Fujishima and K. Honda, "Electrochemical Photolysis of Water at a Semiconductor Electrode," Nature 238, 37-38 (1972).

${ }^{11}$ A. L. Linsebigler, G. Lu, and J. T. Yates, "Photocatalysis on TiO2 Surfaces: Principles, Mechanisms, and Selected Results," Chem. Rev. 95, 735-758 (1995).

${ }^{12}$ S. Overbury, L. Ortiz-Soto, H. Zhu, B. Lee, M. D. Amiridis, and S. Dai, "Comparison of Au Catalysts Supported on Mesoporous Titania and Silica: Investigation of Au Particle Size Effects and Metal-Support Interactions," Catalysis Letters 95, 99-106 (2004).

${ }^{13}$ K. I. Hadjiivanov and D. G. Klissurski, "Surface chemistry of titania (anatase) and titania-supported catalysts," Chem. Soc. Rev. 25, 61-69 (1996).

${ }^{14}$ H. Zhang and J. F. Banfield, "Understanding Polymorphic Phase Transformation Behavior during Growth of Nanocrystalline Aggregates: Insights from TiO2," J. Phys. Chem. B 104, 3481-3487 (2000).

${ }^{15}$ M. R. Ranade, A. Navrotsky, H. Z. Zhang, J. F. Banfield, S. H. Elder, A. Zaban, P. H. Borse, S. K. Kulkarni, G. S. Doran, and H. J. Whitfield, "Energetics of nanocrystalline TiO2," PNAS 99, 6476-6481 (2002).

${ }^{16}$ F. Labat, P. Baranek, C. Domain, C. Minot, and C. Adamo, "Density functional theory analysis of the structural and electronic properties of $\mathrm{TiO} 2 \mathrm{ru}-$ tile and anatase polytypes: Performances of different exchange-correlation functionals," The Journal of Chemical Physics 126, 154703 (2007).

${ }^{17}$ H. Zhang and J. F. Banfield, "Thermodynamic analysis of phase stability of nanocrystalline titania," J. Mater. Chem. 8, 2073-2076 (1998).

${ }^{18}$ J. Aarons, M. Sarwar, D. Thompsett, and C.-K. Skylaris, "Perspective: Methods for large-scale density functional calculations on metallic systems," The Journal of Chemical Physics 145, 220901 (2016).

${ }^{19}$ F. Baletto and R. Ferrando, "Structural properties of nanoclusters: Energetic, thermodynamic, and kinetic effects," Rev. Mod. Phys. 77, 371-423 (2005).

${ }^{20}$ C. Buzea, I. I. Pacheco, and K. Robbie, "Nanomaterials and nanoparticles: Sources and toxicity," Biointerphases 2, MR17-MR71 (2007).

${ }^{21}$ B. Hammer and J. K. Nørskov, "Theoretical surface science and catalysis-calculations and concepts," in Impact of Surface Science on Catalysis, Advances in Catalysis, Vol. 45 (Academic Press, 2000) pp. 71 - 129.

${ }^{22} \mathrm{H}$. Toulhoat and P. Raybaud, "Kinetic interpretation of catalytic activity patterns based on theoretical chemical descriptors," Journal of Catalysis 40th Anniversary Commemorative Issue, 216, 63-72 (2003).

${ }^{23}$ J. K. Nørskov, T. Bligaard, J. Rossmeisl, and C. H. Christensen, "Towards the computational design of solid catalysts," Nature Chemistry 1, 37-46
(2009).

${ }^{24}$ C. J. H. Jacobsen, S. Dahl, B. S. Clausen, S. Bahn, A. Logadottir, and J. K. Nørskov, "Catalyst Design by Interpolation in the Periodic Table: Bimetallic Ammonia Synthesis Catalysts," Journal of the American Chemical Society 123, 8404-8405 (2001).

${ }^{25}$ S. J. Kwon, J. H. Park, K. Y. Koo, W. L. Yoon, and K. B. Yi, "Enhanced catalytic performance of $\mathrm{Pt} / \mathrm{TiO} 2$ catalyst in water gas shift reaction by incorporation of PRGO," Catalysis Today special issue of International Symposium on Catalytic Conversion of Energy and Resources, 293-294, 113-121 (2017).

${ }^{26}$ O. T. Holton and J. W. Stevenson, "The Role of Platinum in Proton Exchange Membrane Fuel Cells," (2013).

${ }^{27}$ L. G. Verga, A. E. Russell, and C.-K. Skylaris, "Ethanol, O, and CO adsorption on Pt nanoparticles: effects of nanoparticle size and graphene support," Physical Chemistry Chemical Physics 20, 25918-25930 (2018).

${ }^{28}$ J. Aarons, L. Jones, A. Varambhia, K. E. MacArthur, D. Ozkaya, M. Sarwar, C.-K. Skylaris, and P. D. Nellist, "Predicting the Oxygen-Binding Properties of Platinum Nanoparticle Ensembles by Combining High-Precision Electron Microscopy and Density Functional Theory," Nano Lett. 17, 4003 4012 (2017).

${ }^{29}$ C.-K. Skylaris, P. D. Haynes, A. A. Mostofi, and M. C. Payne, "Introducing ONETEP: Linear-scaling density functional simulations on parallel computers," The Journal of Chemical Physics 122, 084119 (2005).

${ }^{30}$ S. J. Clark, M. D. Segall, C. J. Pickard, P. J. Hasnip, M. I. J. Probert, K. Refson, and M. C. Payne, "First principles methods using CASTEP," Zeitschrift für Kristallographie - Crystalline Materials 220, 567-570 (2009).

${ }^{31}$ N. Marzari, D. Vanderbilt, and M. C. Payne, "Ensemble DensityFunctional Theory for Ab Initio Molecular Dynamics of Metals and FiniteTemperature Insulators," Phys. Rev. Lett. 79, 1337-1340 (1997).

${ }^{32}$ Á. Ruiz-Serrano and C.-K. Skylaris, "A variational method for density functional theory calculations on metallic systems with thousands of atoms," The Journal of Chemical Physics 139, 054107 (2013).

${ }^{33}$ P. E. Blöchl, "Projector augmented-wave method," Phys. Rev. B 50, 17953 17979 (1994).

${ }^{34}$ K. F. Garrity, J. W. Bennett, K. M. Rabe, and D. Vanderbilt, "Pseudopotentials for high-throughput DFT calculations," Computational Materials Science 81, 446-452 (2014).

${ }^{35}$ J. P. Perdew, K. Burke, and M. Ernzerhof, "Generalized Gradient Approximation Made Simple," Physical Review Letters 77, 3865-3868 (1996).

${ }^{36}$ Y. Zhang and W. Yang, "Comment on "Generalized gradient approximation made simple"," Physical Review Letters 80, 890 (1998).

${ }^{37}$ M. Rezaee, S. M. M. Khoie, and K. H. Liu, "The role of brookite in mechanical activation of anatase-to-rutile transformation of nanocrystalline TiO2: An XRD and Raman spectroscopy investigation," CrystEngComm 13, 5055-5061 (2011).

${ }^{38}$ J. R. Kitchin, J. K. Nørskov, M. A. Barteau, and J. G. Chen, "Modification of the surface electronic and chemical properties of $\mathrm{Pt}(111)$ by subsurface 3d transition metals," J. Chem. Phys. 120, 10240-10246 (2004).

${ }^{39}$ R. J. Nicholls, A. J. Morris, C. J. Pickard, and J. R. Yates, "OptaDOS - a new tool for EELS calculations," J. Phys.: Conf. Ser. 371, 012062 (2012).

${ }^{40}$ J. K. Burdett, T. Hughbanks, G. J. Miller, J. W. Richardson, and J. V. Smith, "Structural-electronic relationships in inorganic solids: powder neutron diffraction studies of the rutile and anatase polymorphs of titanium dioxide at 15 and 295 K," J. Am. Chem. Soc. 109, 3639-3646 (1987).

${ }^{41}$ M. Lazzeri, A. Vittadini, and A. Selloni, "Structure and energetics of stoichiometric $\$\{\backslash \text { mathrm }\{\mathrm{TiO}\}\}_{-}\{2\} \$$ anatase surfaces," Phys. Rev. B 63, 155409 (2001).

${ }^{42}$ A. Vittadini, M. Casarin, and A. Selloni, "Chemistry of and on TiO2anatase surfaces by DFT calculations: a partial review," Theor Chem Account 117, 663-671 (2007).

${ }^{43}$ K. Rossi, T. Ellaby, L. O. Paz-Borbón, I. Atanasov, L. Pavan, and F. Baletto, "Melting of large Pt@MgO( 100$)$ icosahedra," J. Phys.: Condens. Matter 29, 145402 (2017).

${ }^{44}$ W. Tang, E. Sanville, and G. Henkelman, "A grid-based Bader analysis algorithm without lattice bias," J. Phys.: Condens. Matter 21, 084204 (2009).

${ }^{45}$ L. Li, A. H. Larsen, N. A. Romero, V. A. Morozov, C. Glinsvad, F. AbildPedersen, J. Greeley, K. W. Jacobsen, and J. K. Nørskov, "Investigation of Catalytic Finite-Size-Effects of Platinum Metal Clusters," The Journal of Physical Chemistry Letters 4, 222-226 (2013). 
${ }^{46}$ B. Hammer and J. K. Nørskov, "Electronic factors determining the reactivity of metal surfaces," Surface Science 343, 211-220 (1995).

${ }^{47}$ J. Greeley, J. K. Nørskov, and M. Mavrikakis, "Electronic structure and catalysis on metal surfaces," Annu. Rev. Phys. Chem. 53, 319-348 (2002).

${ }^{48}$ A. Vojvodic, J. K. Nørskov, and F. Abild-Pedersen, "Electronic Structure Effects in Transition Metal Surface Chemistry," Top Catal 57, 25-32 (2014).

${ }^{49}$ G. A. Tritsaris, J. Greeley, J. Rossmeisl, and J. K. Nørskov, “Atomic-Scale Modeling of Particle Size Effects for the Oxygen Reduction Reaction on Pt," Catal Lett 141, 909-913 (2011).

${ }^{50}$ P. J. Feibelman, B. Hammer, J. K. Nørskov, F. Wagner, M. Scheffler, R. Stumpf, R. Watwe, and J. Dumesic, “The CO/Pt(111) Puzzle," J. Phys.
Chem. B 105, 4018-4025 (2001).

${ }^{51} \mathrm{M}$. Lynch and P. Hu, "A density functional theory study of $\mathrm{CO}$ and atomic oxygen chemisorption on Pt(111)," Surface Science 458, 1-14 (2000).

${ }^{52}$ J. V. Barth, "Transport of adsorbates at metal surfaces: from thermal migration to hot precursors," Surface Science Reports 40, 75-149 (2000).

${ }^{53}$ J.-X. Liu, I. A. W. Filot, Y. Su, B. Zijlstra, and E. J. M. Hensen, "Optimum Particle Size for Gold-Catalyzed CO Oxidation,” J. Phys. Chem. C 122, 8327-8340 (2018).

${ }^{54}$ L. G. Verga, J. Aarons, M. Sarwar, D. Thompsett, A. E. Russell, and C.K. Skylaris, "Effect of graphene support on large Pt nanoparticles," Phys. Chem. Chem. Phys. 18, 32713-32722 (2016). 